\title{
ESTIMATE OF THE CAUCHY INTEGRAL OVER AHLFORS REGULAR CURVES
}

\author{
MARK MELNIKOV AND XAVIER TOLSA
}

\begin{abstract}
We obtain the complete characterization of those domains $G \subset \mathbb{C}$ which admit the so called estimate of the Cauchy integral, that is to say, $\left|\int_{\partial G} f(z) d z\right| \leq C(G)\|f\|_{\infty} \gamma(E)$ for all $E \subset G$ and $f \in H^{\infty}(G \backslash E)$, where $\gamma(E)$ is the analytic capacity of $E$. The corresponding result for continuous functions $f$ and the continuous analytic capacity $\alpha(E)$ is also proved.
\end{abstract}

\section{Introduction}

The problem of estimating the Cauchy integral over the boundary of a domain was posed by Vitushkin in connection with the theory of uniform rational approximation on compact subsets of the complex plane. The problem consists of characterizing those bounded domains $G \subset \mathbb{C}$ with rectifiable boundary for which there exists a constant $C(G)$ such that for any compact set $E \subset G$ and any function $f$ bounded and holomorphic on $G \backslash E$ the following estimate holds:

$$
\left|\int_{\partial G} f(z) d z\right| \leq C_{1}(G)\|f\|_{\infty} \gamma(E)
$$

where $\gamma(E)$ is the analytic capacity of $E$ (see next section for the precise meaning of the notions appearing in this section). Vitushkin also raised the analogous question for functions $f$ continuous on $\bar{G}$ and holomorphic on $G \backslash E$, changing $\gamma(E)$ by the continuous analytic capacity $\alpha(E)$.

The estimate (1) was proved in 1966 in [Me1] for $G$ being a disk, and more generally, for $G$ with analytic boundary. Later on, Vitushkin [Vi1] proved the estimate (1) for domains $G$ bounded by piecewise Lyapunov curves (i.e. piecewise $\mathcal{C}^{1+\varepsilon}$ curves). In [De], Davie generalized the result to the case of hypolyapunov curves (i.e. curves satisfying a Dini type condition).

In [Vi2, Section III.1], Vitushkin showed an example of a domain $G$ with rectifiable boundary such that the estimate of the integral (1) does not hold (that is, rectifiability of the boundary alone does not imply (1)), and he conjectured that (1) holds if $G$ is a Jordan domain such that

$$
\gamma(F) \geq C_{2}(G) \mathcal{H}^{1}(F) \text { for all closed subsets } F \subset \partial G,
$$

for some constant $C_{2}(G)>0\left(\mathcal{H}^{1}\right.$ denotes the 1-dimensional Hausdorff measure, or arc length). In the present paper we prove that this conjecture is true.

Let us remark that when $\partial G$ is a curve, condition (2) is equivalent to the fact $\partial G$ is Ahlfors regular, that is to say,

$$
\mathcal{H}^{1}(B(x, r) \cap \partial G) \leq C r \quad \text { for all } x \in \mathbb{C}, r>0 .
$$

The authors were partially supported by grants BFM2000-0361, HPRN-2000-0116, and 2001SGR-00431. 
This follows from a theorem of David [Dd] which asserts that the Cauchy integral operator is bounded in $L^{2}$ on Ahlfors regular curves.

Now we state our result in detail.

Theorem 1. Let $G$ be a bounded open set in $\mathbb{C}$ whose boundary $\partial G$ is a finite disjoint union of Jordan rectifiable (closed) curves. Then, the following conditions are equivalent:

(a) There exists some constant $C_{2}(G)>0$ such that for any closed set $F \subset \partial G$,

$$
\gamma(F) \geq C_{2}(G) \mathcal{H}^{1}(F) .
$$

(b) The Cauchy integral operator $\mathcal{C}_{\mathcal{H}^{1} \mid \partial G}$ is bounded on $L^{2}\left(\mathcal{H}_{\mid \partial G}^{1}\right)$.

(c) There exists some constant $C_{1}(G)$ such that for any compact set $E \subset G$ and any function $f \in H^{\infty}(G \backslash E)$, we have

$$
\left|\int_{\partial G} f(z) d z\right| \leq C_{1}(G)\|f\|_{\infty} \gamma(E)
$$

(d) There exists some constant $C_{3}(G)$ such that for any compact set $E \subset G$ and any function $f \in H^{\infty}(G \backslash E) \cap C(\bar{G})$, we have

$$
\left|\int_{\partial G} f(z) d z\right| \leq C_{3}(G)\|f\|_{\infty} \alpha(E)
$$

The constants $C_{1}(G)$ and $C_{3}(G)$ depend only on $C_{2}(G)$, or equivalently, on the $L^{2}\left(\mathcal{H}_{\mid \partial G}^{1}\right)$ norm of $\mathcal{C}_{\mathcal{H}^{1} \mid \partial G}$, and conversely.

Notice that in Theorem 1 we consider a more general setting than $G$ being a Jordan domain. Moreover, we show that condition (2) is not only sufficient, but also necessary for the estimate of the Cauchy integral.

Vitushkin's original motivation for studying estimates like (1) was to obtain necessary and sufficient conditions for uniform rational approximation. Let $X \subset \mathbb{C}$ be compact and, as usual, let $R(X)$ be the algebra of uniform on $X$ limits of rational functions with poles out of $X$, and $A(X)$ the algebra of functions continuous on $X$ and holomorphic on $\stackrel{\circ}{X}$ (this is the interior of $X$ ). Given $f \in A(X)$ one asks when $f$ also belongs to $R(X)$. A direct consequence of Theorem 1 is that such function $f$ has to satisfy the following estimate, for any Jordan domain $G$ with Ahlfors regular boundary:

$$
\left|\int_{\partial G} f(z) d z\right| \leq C(G) \omega_{f}(\operatorname{diam}(G)) \gamma(G \backslash X)
$$

where $C(G)$ depends only on the Ahlfors regularity constant of $\partial G$ and $\omega_{f}(\cdot)$ is the modulus of continuity of $f$. Conversely, condition (5) is also sufficient for $f$ to be in $R(X)$. Indeed, it is enough that (5) holds either for all the squares $G \subset \mathbb{C}$ [Vi2] or for all the disks $G \subset \mathbb{C}[\mathrm{Pa}]$.

In the proof of Theorem 1 we use the quite recent results and ideas involved in the proof of the semiadditivity of $\gamma$ [To4] and $\alpha$ [To5].

The plan of the paper is the following. In Section 2 we recall some preliminary definitions and results. In Section 3 we prove the equivalence $(\mathrm{a}) \Leftrightarrow(\mathrm{b})$ of Theorem 1. The main implications are $(\mathrm{b}) \Rightarrow(\mathrm{c})$ and $(\mathrm{b}) \Rightarrow(\mathrm{d})$, and they are proved in Sections 4 and 5 respectively. In Section 6 we show that (c) $\Rightarrow$ (a) and, finally, in Section 7 that $(\mathrm{d}) \Rightarrow(\mathrm{a})$. 


\section{Preliminaries}

Throughout all the paper, the letter $C$ will stand for an absolute constant that may change at different occurrences. Constants with subscripts, such as $C_{1}$, will retain its value, in general.

The notation $A \approx B$ means that there exists an absolute constant $C>0$ such that $C^{-1} A \leq B \leq C A$.

\subsection{Analytic capacity, the Cauchy transform, and curvature of measures.} The analytic capacity of a compact set $E \subset \mathbb{C}$ is

$$
\gamma(E)=\sup \left|f^{\prime}(\infty)\right|,
$$

where the supremum is taken over all holomorphic functions $f: \mathbb{C} \backslash E \longrightarrow \mathbb{C}$ with $|f| \leq 1$ on $\mathbb{C} \backslash E$, and $f^{\prime}(\infty)=\lim _{z \rightarrow \infty} z(f(z)-f(\infty))$.

The continuous analytic capacity of $E$ is

$$
\alpha(E)=\sup \left|f^{\prime}(\infty)\right|,
$$

where the supremum is taken over all complex functions which are continuous in $\mathbb{C}$, holomorphic on $\mathbb{C} \backslash E$, and satisfy $|f(z)| \leq 1$ for all $z \in \mathbb{C}$.

A positive Radon measure $\mu$ is said to have linear growth if there exists some constant $C$ such that $\mu(B(x, r)) \leq C r$ for all $x \in \mathbb{C}, r>0$. The linear density of $\mu$ at $x \in \mathbb{C}$ is (if it exists)

$$
\Theta_{\mu}(x)=\lim _{r \rightarrow 0} \frac{\mu(B(x, r))}{r} .
$$

Given a complex Radon measure $\nu$ on $\mathbb{C}$, the Cauchy transform of $\nu$ is

$$
\mathcal{C} \nu(z)=\int \frac{1}{\xi-z} d \nu(\xi)
$$

This definition does not make sense, in general, for $z \in \operatorname{supp}(\nu)$, although one can easily see that the integral above is convergent at a.e. $z \in \mathbb{C}$ (with respect to Lebesgue measure). This is the reason why one considers the truncated Cauchy transform of $\nu$, which is defined as

$$
\mathcal{C}_{\varepsilon} \nu(z)=\int_{|\xi-z|>\varepsilon} \frac{1}{\xi-z} d \nu(\xi),
$$

for any $\varepsilon>0$ and $z \in \mathbb{C}$. Given a $\mu$-measurable function $f$ on $\mathbb{C}$ (where $\mu$ is some fixed positive Radon measure on $\mathbb{C}$ ), the Cauchy integral operator $\mathcal{C}_{\mu}$ is defined by

$$
\mathcal{C}_{\mu} f:=\mathcal{C}(f d \mu)
$$

The $\varepsilon$-truncated version of $\mathcal{C}_{\mu}$ is $\mathcal{C}_{\mu, \varepsilon} f:=\mathcal{C}_{\varepsilon}(f d \mu)$. We say the Cauchy integral operator is bounded on $L^{2}(\mu)$ if the operators $\mathcal{C}_{\mu, \varepsilon}$ are bounded on $L^{2}(\mu)$ uniformly on $\varepsilon>0$.

The maximal Cauchy transform of a complex measure $\nu$ is

$$
\mathcal{C}_{*} \nu(x)=\sup _{\varepsilon>0}\left|\mathcal{C}_{\varepsilon} \nu(x)\right| .
$$

We also set $\mathcal{C}_{\mu, *}(f)=\mathcal{C}_{*}(f d \mu)$.

If in the supremum (6) which defines $\gamma(E)$, additionally we ask the functions $f$ to be Cauchy transforms of positive measures, we get the capacity $\gamma_{+}$of $E$. The definition of $\alpha_{+}$is analogous. 
Given three pairwise different points $x, y, z \in \mathbb{C}$, their Menger curvature is

$$
c(x, y, z)=\frac{1}{R(x, y, z)},
$$

where $R(x, y, z)$ is the radius of the circumference passing through $x, y, z$ (with $R(x, y, z)=\infty, c(x, y, z)=0$ if $x, y, z$ lie on a same line). If two among these points coincide, we let $c(x, y, z)=0$. For a positive Radon measure $\mu$, we set

$$
c_{\mu}^{2}(x)=\iint c(x, y, z)^{2} d \mu(y) d \mu(z),
$$

and we define the curvature of $\mu$ as

$$
c^{2}(\mu)=\int c_{\mu}^{2}(x) d \mu(x)=\iiint c(x, y, z)^{2} d \mu(x) d \mu(y) d \mu(z) .
$$

The notion of curvature of measures was introduced in [Me2], where some estimates of analytic capacity in terms of curvature were obtained.

Curvature of measures is connected to the Cauchy transform too. Indeed, Melnikov and Verdera $[\mathrm{MV}]$ proved that if $\mu$ has linear growth, then

$$
\left\|\mathcal{C}_{\varepsilon} \mu\right\|_{L^{2}(\mu)}^{2}=\frac{1}{6} c_{\varepsilon}^{2}(\mu)+O(\mu(\mathbb{C})),
$$

where $c_{\varepsilon}^{2}(\mu)$ is an $\varepsilon$-truncated version of $c^{2}(\mu)$ (defined as in the right hand side of (7), but with the triple integral over $\{x, y, z \in \mathbb{C}:|x-y|,|y-z|,|x-z|>\varepsilon\})$.

In [To4], the following result has been proved:

Theorem A. For any compact set E, we have

(9)

$$
\begin{aligned}
& \gamma(E) \approx \gamma_{+}(E) \\
& \approx \sup \left\{\mu(E): \operatorname{supp}(\mu) \subset E, \mu(B(x, r)) \leq r \forall x \in E, r>0 \text { and } c^{2}(\mu) \leq \mu(E)\right\} \\
& \approx \sup \{\mu(E): \operatorname{supp}(\mu) \subset E, \mu(B(x, r)) \leq r \forall x \in E, r>0 \text { and } \\
&\left.\quad\left\|\mathcal{C}_{\mu}\right\|_{L^{2}(\mu), L^{2}(\mu)} \leq 1\right\},
\end{aligned}
$$

with absolute constants.

The corresponding result for $\alpha$ was obtained in [To5].

Theorem B. For any compact set E, we have

$$
\begin{aligned}
& \alpha(E) \approx \alpha_{+}(E) \\
& \approx \sup \left\{\mu(E): \operatorname{supp}(\mu) \subset E, \Theta_{\mu}(x)=0 \forall x \in E,\right. \\
& \left.\mu(B(x, r)) \leq r \forall x \in E, r>0 \text { and } c^{2}(\mu) \leq \mu(E)\right\} \\
& \approx \sup \left\{\mu(E): \operatorname{supp}(\mu) \subset E, \Theta_{\mu}(x)=0 \forall x \in E,\right. \\
& \left.\mu(B(x, r)) \leq r \forall x \in E, r>0 \text { and }\left\|\mathcal{C}_{\mu}\right\|_{L^{2}(\mu), L^{2}(\mu)} \leq 1\right\},
\end{aligned}
$$

with absolute constants. 
A direct consequence of the Theorems A and B is that $\gamma$ and $\alpha$ are semiadditive. That is, for all compact sets $E, F \subset \mathbb{C}$,

$$
\gamma(E \cup F) \leq C(\gamma(E)+\gamma(F)),
$$

and

$$
\alpha(E \cup F) \leq C(\alpha(E)+\alpha(F)) .
$$

The following potential was introduced by Verdera in [Ve]:

$$
U_{\mu}(x):=M \mu(x)+c_{\mu}(x),
$$

where $M$ is the maximal radial Hardy-Littlewood operator:

$$
M \mu(x)=\sup _{r>0} \frac{\mu(B(x, r))}{r},
$$

and $c_{\mu}(x)=\left(c_{\mu}^{2}(x)\right)^{1 / 2}$. We remark that $\gamma$ can be characterized in terms of this potential:

$$
\gamma(E) \approx \sup \left\{\mu(E): \operatorname{supp}(\mu) \subset E, U_{\mu}(x) \leq 1 \forall x \in \mathbb{C}\right\} .
$$

An analogous characterization for $\alpha$ and $\alpha_{+}$exists. See [To5] for the details.

2.2. Vitushkin's localization operator $V_{\varphi}$. Given $f \in L_{l o c}^{1}(\mathbb{C})$ and $\varphi \in C^{\infty}$ compactly supported, we set

$$
V_{\varphi} f:=\varphi f-\frac{1}{\pi} \mathcal{C}(f \bar{\partial} \varphi)=\frac{1}{\pi} \mathcal{C}(\varphi \bar{\partial} f) .
$$

Here $\bar{\partial} f$ should be understood in the sense of distributions. Recall that if the support $\varphi$ is contained in a ball of radius $r,\|\varphi\|_{\infty} \leq C_{4}$, and $\|\nabla \varphi\|_{\infty} \leq C_{4} / r$, then $V_{\varphi} f$ has the following properties (see [Ga, Lemma VIII-7.1], for example):

- $\left\|V_{\varphi} f\right\|_{\infty} \leq C_{5}\|f\|_{\infty}$,

- $\left\|V_{\varphi} f\right\|_{\infty} \leq C_{6} \omega_{f}(r)$, where $\omega_{f}$ stands for the modulus of continuity of $f$,

- $V_{\varphi} f$ is holomorphic outside $\operatorname{supp}(\bar{\partial} f) \cap \operatorname{supp}(\varphi)$,

- if $f$ is continuous on $\mathbb{C}$, then $V_{\varphi} f$ is also continuous in $\mathbb{C}$.

The constants $C_{5}$ and $C_{6}$ depend only on $C_{4}$.

\section{Proof of the equivalence (a) $\Leftrightarrow$ (b)}

The implication (b) $\Rightarrow$ (a) is well known. It follows from the fact that the $L^{2}$ boundedness of the Cauchy integral operator implies that it is of weak type $(1,1)$ (see [NTV1] or [To2], for example), and from a dualization of the weak $(1,1)$ inequality. It can also be proved using the estimate of analytic capacity in terms of curvature obtained in [Me2].

We consider now the other implication. So we assume that

$$
\gamma(F) \geq C^{-1} \mathcal{H}^{1}(F) \quad \text { for all closed sets } F \subset \partial G .
$$

This condition implies that $\mathcal{H}_{\mid \partial G}^{1}$ has linear growth, because for any closed ball $\bar{B}(x, r)$ we have

$$
\mathcal{H}^{1}(\bar{B}(x, r) \cap \partial G) \leq C \gamma(\bar{B}(x, r) \cap \partial G) \leq C r .
$$

In [To3] it has been shown that there is an absolute constant $C_{7}$ such that for any complex Radon measure $\nu$ and any $\lambda>0$ the following holds:

$$
\gamma_{+}\left\{x \in \mathbb{C}: \mathcal{C}_{*} \nu(x)>\lambda\right\} \leq C_{7} \frac{\|\nu\|}{\lambda} .
$$


Then, by (12) and the comparability between $\gamma$ and $\gamma_{+}$, we get

$$
\mathcal{H}^{1}\left\{x \in \partial G: \mathcal{C}_{*} \nu(x)>\lambda\right\} \leq C \gamma\left\{x \in \partial G: \mathcal{C}_{*} \nu(x)>\lambda\right\} \leq C \frac{\|\nu\|}{\lambda} .
$$

Thus the Cauchy integral operator is bounded from the space of complex Radon measures $M(\mathbb{C})$ into $L^{1, \infty}\left(\mathcal{H}_{\mid \partial G}^{1}\right)$. In particular, it is of weak type $(1,1)$ (with respect to arc length measure on $\partial G$ ). This is equivalent to the $L^{2}$ boundedness on $\partial G$ (see [To1] or [NTV1], for example).

Let us remark that one can prove the implication $(\mathrm{a}) \Rightarrow(\mathrm{b})$ by different arguments. For example, instead of using the inequality (13), one can use the local $T(b)$ theorem of Nazarov, Treil and Volberg [NTV2].

\section{Proof of the implication (b) $\Rightarrow$ (c)}

4.1. A preliminary lemma. The following result will play a key role in the proof of $(\mathrm{b}) \Rightarrow(\mathrm{c})$.

Lemma 2. Let $E \subset \mathbb{C}$ be compact. There exists an open set $\Omega$ containing $E$ with a Whitney decomposition $\Omega=\bigcup_{i \in I} Q_{i}$, where $\left\{Q_{i}\right\}_{i \in I}$ are Whitney squares such that $\gamma_{+}(\Omega) \approx \gamma_{+}(E)$ and

$$
\sum_{i \in I} \gamma_{+}\left(E \cap 2 Q_{i}\right) \leq C \gamma_{+}(E)
$$

Let us remark that, unless stated otherwise, we assume that all the squares are closed and have sides parallel to the axes.

Proof. In (a) and (b) in Lemma 5.1 of [To4] the same result has been proved assuming that $E$ is a finite union of segments. To prove it for this type of sets, it has been shown in [To4, Lemma 4.1] that there exists a measure $\sigma$ supported on $E$, with linear growth, such that $\sigma(E) \approx \gamma_{+}(E)$ and $U_{\sigma}(x) \geq 1$ for all $x \in E$. If $E$ is an arbitrary compact set, then we have

$$
\gamma_{+}(E) \approx \inf \left\{\mu(\mathbb{C}): \mu \in M_{+}(\mathbb{C}), U_{\mu}(x) \geq 1 \forall x \in E\right\},
$$

where $M_{+}(\mathbb{C})$ stands for the set of all positive Radon measures, by [To3, Theorem 3.3]. As a consequence, in this case there also exists some measure $\sigma$ (which in general will not be supported on $E$ and will not have linear growth) such that $\sigma(E) \approx \gamma_{+}(E)$ and $U_{\sigma}(x) \geq 1$ for all $x \in E$.

Now the same arguments used to prove (a) and (b) of [To4, Lemma 5.1] work since it can be checked that the assumptions concerning the support and linear growth of $\sigma$ are not necessary.

4.2. Proof of the implication $(\mathbf{b}) \Rightarrow(\mathbf{c})$. Suppose that the Cauchy integral operator $\mathcal{C}_{\mathcal{H}_{\mid \partial G}^{1}}$ is bounded on $L^{2}\left(\mathcal{H}_{\mid \partial G}^{1}\right)$. We want to show that $(3)$ holds. We assume that $f$ vanishes on $\mathbb{C} \backslash \bar{G}$ and also, by homogeneity, that $\|f\|_{\infty} \leq 1$.

Let $\Omega$ be the open set containing $E$ in Lemma 2 , and $\Omega=\bigcup_{i \in I} Q_{i}$ a Whitney decomposition into squares satisfying the conditions mentioned in the same lemma. Now we consider a partition of unity: let $\left\{\varphi_{i}\right\}_{i \in I}$ be a family of $C^{\infty}$ functions such that $0 \leq \varphi_{i} \leq 1,\left\|\nabla \varphi_{i}\right\|_{\infty} \leq C / \ell\left(Q_{i}\right)$ and $\operatorname{supp}\left(\varphi_{i}\right) \subset \frac{3}{2} Q_{i}$ for each $i \in I$, so that $\sum_{i \in I} \varphi_{i}=1$ on $\Omega$. 
Consider the (finite) subfamily of squares $\left\{Q_{j}\right\}_{j \in J}, J \subset I$, such that $2 Q_{j} \cap E \neq \varnothing$. Notice that $\psi:=\sum_{j \in J} \varphi_{j}=1$ on a neighborhood of $E$. Moreover, $\psi$ is a compactly supported $C^{\infty}$ function because $J$ is finite. Then we have

$$
f=\sum_{j \in J} V_{\varphi_{j}} f+V_{1-\psi} f .
$$

The function $V_{1-\psi} f$ is bounded on $G$, although its $L^{\infty}$ norm may depend on \#J. Also, it is holomorphic in $G$ because

$$
\bar{\partial}\left(V_{1-\psi} f\right)=(1-\psi) \bar{\partial} f=0 \quad \text { in } G,
$$

since $1-\psi$ vanishes in a neighborhood of $E$ and $f$ is holomorphic in $G \backslash E$. Thus,

$$
\int_{\partial G} f(z) d z=\sum_{j \in J} \int_{\partial G} V_{\varphi_{j}} f(z) d z .
$$

Let $J_{1} \subset J$ be the set of indices such that $2 Q_{j} \cap \partial G=\varnothing$ if $j \in J_{1}$, and $J_{2}=J \backslash J_{1}$. By the definition of $\gamma$, for $j \in J_{1}$ we have

$$
\left|\int_{\partial G} V_{\varphi_{j}} f(z) d z\right| \leq C \gamma\left(E \cap 2 Q_{j}\right)
$$

because $E \cap 2 Q_{i} \subset G$ and $\left\|V_{\varphi_{j}} f\right\|_{\infty} \leq C$. Therefore, using Lemma 2 and the fact that $\gamma \approx \gamma_{+}$, we obtain

$$
\left|\sum_{j \in J_{1}} \int_{\partial G} V_{\varphi_{j}} f(z) d z\right| \leq C \sum_{j \in J_{1}} \gamma\left(E \cap 2 Q_{j}\right) \leq C \gamma(E) .
$$

For $j \in J_{2}$ we will show below that

$$
\left|\int_{\partial G} V_{\varphi_{j}} f(z) d z\right| \leq C\left(\gamma\left(E \cap 2 Q_{j}\right)+\mathcal{H}^{1}\left(\partial G \cap 3 Q_{j}\right)\right) .
$$

Before proving this estimate, let us see that (3) follows from (14), (15), and (16). Indeed, using Lemma 2 again, by the finite overlap of the Whitney squares $Q_{j}$, we get

$$
\begin{aligned}
\left|\sum_{j \in J_{2}} \int_{\partial G} V_{\varphi_{j}} f(z) d z\right| & \leq C\left(\sum_{j \in J} \gamma\left(E \cap 2 Q_{j}\right)+\sum_{j \in J} \mathcal{H}^{1}\left(\partial G \cap 3 Q_{j}\right)\right) \\
& \leq C\left(\gamma(E)+\mathcal{H}^{1}(\partial G \cap \Omega)\right) .
\end{aligned}
$$

Because of the $L^{2}\left(\mathcal{H}_{\mid \partial G}^{1}\right)$ boundedness of the Cauchy integral operator, we have

$$
\mathcal{H}^{1}(\partial G \cap \Omega) \leq C \gamma_{+}(\partial G \cap \Omega) \leq C \gamma_{+}(\Omega) \leq C \gamma_{+}(E) .
$$

Thus,

$$
\left|\sum_{j \in J_{2}} \int_{\partial G} V_{\varphi_{j}} f(z) d z\right| \leq C \gamma(E)
$$

which, jointly with (15), yields (3).

It only remains to prove (16) for $j \in J_{2}$. Let $D_{j}$ be a disk with radius $\ell\left(Q_{j}\right) / 4$ whose center $z_{j}$ coincides with the center of $Q_{j}$. Consider the following measure:

$$
\nu_{j}:=\frac{-1}{\pi \mathcal{H}^{2}\left(D_{j}\right)}\left(\int f \bar{\partial} \varphi_{j} d m\right) \mathcal{H}^{2} \mid D_{j} .
$$


We want to compare $V_{\varphi_{j}} f$ with the function $g_{j}:=\mathcal{C} \nu_{j}$. Notice that

$$
\frac{1}{\pi} \int f \bar{\partial} \varphi_{j} d m=\left(V_{\varphi_{j}} f\right)^{\prime}(\infty)=g_{j}^{\prime}(\infty) .
$$

Then, using the definition of $\gamma$, the fact that $\left\|V_{\varphi_{j}} f\right\|_{\infty} \leq C$, and the semiadditivity of $\gamma$, we get

$$
\begin{aligned}
\left|\frac{1}{\pi} \int f \bar{\partial} \varphi_{j} d m\right| & \leq C \gamma\left((E \cup \partial G) \cap 2 Q_{j}\right) \leq C\left(\gamma\left(E \cap 2 Q_{j}\right)+\gamma\left(\partial G \cap 2 Q_{j}\right)\right) \\
& \leq C\left(\gamma\left(E \cap 2 Q_{j}\right)+\mathcal{H}^{1}\left(\partial G \cap 2 Q_{j}\right)\right) .
\end{aligned}
$$

As a consequence,

$$
\left\|g_{j}\right\|_{\infty} \leq\left|\frac{C}{\ell\left(Q_{j}\right)} \int f \bar{\partial} \varphi_{j} d m\right| \leq C \frac{\gamma\left(E \cap 2 Q_{j}\right)+\mathcal{H}^{1}\left(\partial G \cap 2 Q_{j}\right)}{\ell\left(Q_{j}\right)} \leq C .
$$

In the last inequality we used the fact that the arc length measure on $\partial G$ has linear growth, because the Cauchy integral operator is bounded on $L^{2}\left(\mathcal{H}_{\mid \partial G}^{1}\right)$. Since $g_{j}$ and $V_{\varphi_{j}} f$ are bounded by $C$ and $\left(V_{\varphi_{j}} f\right)^{\prime}(\infty)=g_{j}^{\prime}(\infty)$, the following estimate holds for $z \notin 3 Q_{j}$ :

$$
\begin{aligned}
\left|V_{\varphi_{j}} f(z)-g_{j}(z)\right| & \leq \frac{C \ell\left(2 Q_{j}\right) \gamma\left((E \cup \partial G) \cap 2 Q_{j}\right)}{\operatorname{dist}\left(z, 2 Q_{j}\right)^{2}} \\
& \leq \frac{C \ell\left(Q_{j}\right)\left[\gamma\left(E \cap 2 Q_{j}\right)+\mathcal{H}^{1}\left(\partial G \cap 2 Q_{j}\right)\right]}{\left|z-z_{j}\right|^{2}} .
\end{aligned}
$$

Let us estimate the integral

$$
\int_{\partial G}\left|V_{\varphi_{j}} f(z)-g_{j}(z)\right| d \mathcal{H}^{1}(z)=\int_{\partial G \cap 3 Q_{j}}+\int_{\partial G \backslash 3 Q_{j}}=: I_{1}+I_{2} .
$$

From the uniform boundedness of $g_{j}$ and $V_{\varphi_{j}} f$, it follows immediately that $I_{1} \leq$ $C \mathcal{H}^{1}\left(\partial G \cap 3 Q_{j}\right)$. To deal with $I_{2}$ we use (19) and the fact that the arc length measure on $\partial G$ has linear growth. Then we get

$$
\begin{aligned}
I_{2} & \leq \int_{\partial G \backslash 3 Q_{j}} \frac{C \ell\left(Q_{j}\right)\left[\gamma\left(E \cap 2 Q_{j}\right)+\mathcal{H}^{1}\left(\partial G \cap 2 Q_{j}\right)\right]}{\left|z-z_{j}\right|^{2}} d \mathcal{H}^{1}(z) \\
& \leq C\left(\gamma\left(E \cap 2 Q_{j}\right)+\mathcal{H}^{1}\left(\partial G \cap 2 Q_{j}\right)\right) .
\end{aligned}
$$

As a consequence,

$$
\left|\int_{\partial G} V_{\varphi_{j}} f(z) d z\right| \leq C\left(\gamma\left(E \cap 2 Q_{j}\right)+\mathcal{H}^{1}\left(\partial G \cap 3 Q_{j}\right)\right)+\left|\int_{\partial G} g_{j}(z) d z\right| .
$$

By Fubini and (17) we obtain

$$
\left|\int_{\partial G} g_{j}(z) d z\right|=\left|\int_{\partial G} \mathcal{C} \nu_{j}(z) d z\right|=C\left|\nu_{j}(G)\right| \leq C\left(\gamma\left(E \cap 2 Q_{j}\right)+\mathcal{H}^{1}\left(\partial G \cap 2 Q_{j}\right)\right) .
$$

So (16) holds, and the theorem follows. 


\section{Proof of the implication (b) $\Rightarrow$ (d)}

5.1. The capacities $\gamma^{h}$. The capacity $\gamma^{h}$ of a compact set $E \subset \mathbb{C}$, introduced in [To5], is defined as follows. We consider a continuous function $h:(0,+\infty) \rightarrow$ $(0,+\infty)$ such that $h(r) / r$ is non decreasing in $r$,

$$
h(r) \leq r \quad \text { and } \quad h(2 r) \leq 4 h(r) \quad \text { for all } r>0,
$$

and moreover

$$
\lim _{r \rightarrow 0+} \frac{h(r)}{r}=0
$$

We set

$$
\gamma^{h}(E)=\sup \left|f^{\prime}(\infty)\right|
$$

where the supremum is taken over all functions $f \in L^{\infty}(\mathbb{C})$ which are holomorphic in $\mathbb{C} \backslash E$, with $f(\infty)=0,\|f\|_{L^{\infty}(\mathbb{C})} \leq 1$, such that

$$
\left|\int f \bar{\partial} \varphi d \mathcal{L}^{2}\right| \leq h(r) r\|\nabla \varphi\|_{\infty}
$$

for any real function $\varphi \in \mathcal{C}_{c}^{\infty}$ supported on some ball of radius $r$. If $f$ satisfies all these properties we say that $f$ is admissible for $\gamma^{h}$ and $E$, and we write $f \in A^{h}(E)$.

The capacity $\gamma_{+}^{h}(E)$ is defined in an analogous way, but we ask an additional condition on the functions in the supremum above. Namely, $f$ should be the Cauchy transform of some positive Radon measure supported on $E$.

Let us remark that the doubling property $h(2 r) \leq 4 h(r)$, for $r>0$, implies that

$$
h(\lambda t) \leq 4 \lambda^{2} h(t) \quad \text { for all } \lambda>1 \text { and } t>0 .
$$

In [To5], the following result has been proved:

Theorem C. For any compact set $E$, we have

$$
\alpha(E) \approx \sup _{h} \gamma^{h}(E),
$$

with the supremum over all continuous functions $h:(0,+\infty) \longrightarrow(0,+\infty)$ satisfying (20) and (21), with $h(r) / r$ non decreasing. Moreover, for any fixed $h$ fulfilling these properties,

$$
\begin{aligned}
& \gamma^{h}(E) \approx \gamma_{+}^{h}(E) \\
& \approx \sup \left\{\mu(E): \operatorname{supp}(\mu) \subset E, \mu(B(x, r)) \leq h(r) \forall x \in E, r>0, \text { and } c^{2}(\mu) \leq \mu(E)\right\} \\
& \approx \sup \{\mu(E): \operatorname{supp}(\mu) \subset E, \mu(B(x, r)) \leq h(r) \forall x \in E, r>0, \text { and } \\
& \left.\quad\left\|\mathcal{C}_{\mu}\right\|_{L^{2}(\mu), L^{2}(\mu)} \leq 1\right\},
\end{aligned}
$$

with absolute constants (independent of $E$ and $h$, in particular). As a consequence, $\gamma^{h}$ and $\alpha$ are countably semiadditive.

The next lemma asserts that the localization operator $V_{\varphi}$ behaves well with respect to the capacity $\gamma^{h}$ :

Lemma $3\left(\left[\right.\right.$ To5]). Let $E \subset \mathbb{C}$ be compact and $f \in A^{h}(E)$. Let $\varphi$ be a $C^{\infty}$ function supported on $\bar{B}\left(x_{0}, r\right)$, such that $\|\varphi\|_{\infty} \leq C_{8}$ and $\|\nabla \varphi\|_{\infty} \leq C_{8} r^{-1}$. Then there exists some constant $C$ depending on $C_{8}$ such that $C^{-1} V_{\varphi} f \in A^{h}\left(E \cap \bar{B}\left(x_{0}, r\right)\right)$. 
5.2. Estimate of the Cauchy integral in terms of $\gamma^{h}$. We will prove the following result, which may have some independent interest.

Theorem 4. Let $G$ be a bounded open set in $\mathbb{C}$ such that its boundary $\partial G$ is a finite disjoint union of Jordan rectifiable curves. Suppose that the Cauchy integral operator $\mathcal{C}_{\mathcal{H}^{1} \mid \partial G}$ is bounded on $L^{2}\left(\mathcal{H}_{\mid \partial G}^{1}\right)$. Then there exists a constant $C_{9}(G)$, which only depends on the $L^{2}\left(\mathcal{H}_{\mid \partial G}^{1}\right)$ norm of $\mathcal{C}_{\mathcal{H}^{1} \mid \partial G}$, such that for any compact set $E \subset \mathbb{C}$ and any function $f \in C(\overline{\mathbb{C}}) \cap H^{\infty}(G \backslash E)$, we have

$$
\left|\int_{\partial G} f(z) d z\right| \leq C_{9}(G) \gamma^{h}(E)
$$

where $h(r)=r \omega_{f}(r)$.

The implication $(\mathrm{b}) \Rightarrow(\mathrm{d})$ in Theorem 1 follows easily from the preceding theorem. Indeed, given $f \in \mathcal{C}(\bar{G}) \cap H^{\infty}(G \backslash E)$, we may extend it continuously to the whole complex plane without increasing $\|f\|_{\infty}$. Then we apply Theorem 4 to $f /\|f\|_{\infty}$, and we get

$$
\left|\int_{\partial G} f(z) d z\right| \leq C_{9}(G)\|f\|_{\infty} \gamma^{h}(E) \leq C\|f\|_{\infty} \alpha(E)
$$

(observe that $C_{9}(G)$ does not depend on $h$ ).

Let us remark that the condition $f \in C(\overline{\mathbb{C}}) \cap H^{\infty}(G \backslash E)$, implies that $C^{-1} f \in$ $A^{h}(E)$ for some absolute constant $C$ and $h(r)=r \omega_{f}(r)$ (see [To5, Lemma 4.1]. The converse implication seems to be false in general (as far as we know).

5.3. Preliminary lemmas for the proof of Theorem 4. The following lemma will play an essential role in the proof of Theorem 4 .

Lemma 5. Let $E \subset \mathbb{C}$ be compact. There exists an open set $\Omega$ containing $E$ with a Whitney decomposition $\Omega=\bigcup_{i \in I} Q_{i}$, where $\left\{Q_{i}\right\}_{i \in I}$ are Whitney squares such that $\gamma_{+}^{h}(\Omega) \approx \gamma_{+}^{h}(E)$ and

$$
\sum_{i \in I} \gamma_{+}^{h}\left(E \cap 2 Q_{i}\right) \leq C \gamma_{+}^{h}(E) .
$$

The proof of this result is similar to the one of Lemma 2. See Lemma 7.2 of [To5] for the details.

We will also need next lemma.

Lemma 6. Consider $f \in C(\overline{\mathbb{C}})$ and set $h(r)=r \omega_{f}(r)$. Let $\varphi$ be a $C^{\infty}$ function supported on a square $Q$ such that $\|\varphi\|_{\infty} \leq 1$ and $\|\nabla \varphi\|_{\infty} \leq C_{10} / \ell(Q)$. Let $\Gamma$ be an Ahlfors regular closed Jordan curve which intersects $Q$. We have

$$
\left|\int_{\Gamma} V_{\varphi} f(z) d z\right| \leq C_{11} h(\operatorname{diam}(\Gamma \cap 2 Q)),
$$

where $C_{11}$ only depends on $C_{10}$ and the Ahlfors regularity constant of $\Gamma$.

Proof. We consider first the case $\Gamma \subset 2 Q$. We take $z_{0} \in \Gamma$. Notice that $V_{\varphi} f=$ $V_{\varphi}\left(f-f\left(z_{0}\right)\right)$, because $V_{\varphi} 1=0$. So if we set $\widetilde{f}(z):=f(z)-f\left(z_{0}\right)$, we have

$$
\int_{\Gamma} V_{\varphi} f(z) d z=\int_{\Gamma} V_{\varphi} \widetilde{f}(z) d z=\int_{\Gamma} \varphi(z) \widetilde{f}(z) d z-\frac{1}{\pi} \int_{\Gamma} \mathcal{C}(\widetilde{f} \bar{\partial} \varphi)(z) d z=: I_{1}+I_{2} .
$$


First we estimate $I_{1}$ :

$$
\begin{aligned}
\left|I_{1}\right| & \leq \int_{\Gamma}|\varphi(z) \widetilde{f}(z)| d \mathcal{H}^{1}(z) \leq\|\widetilde{f}\|_{\infty, \Gamma} \mathcal{H}^{1}(\Gamma) \\
& \leq C \omega_{f}(\operatorname{diam}(\Gamma)) \mathcal{H}^{1}(\Gamma) \leq C h(\operatorname{diam}(\Gamma)) .
\end{aligned}
$$

Now we turn our attention to $I_{2}$. Observe that by Cauchy's formula we have

$$
I_{2}=C \int_{\operatorname{Int}(\Gamma)} \tilde{f}(z) \bar{\partial} \varphi(z) d \mathcal{H}^{2}(z)
$$

where Int $(\Gamma)$ stands for the bounded component of $\mathbb{C} \backslash \Gamma$. Then we get

$$
\left|I_{2}\right| \leq \frac{C}{\ell(Q)}\|\widetilde{f}\|_{\infty, \operatorname{Int}(\Gamma)} \mathcal{H}^{2}(\operatorname{Int}(\Gamma))^{2} \leq \frac{C \omega_{f}(\operatorname{diam}(\Gamma)) \operatorname{diam}(\Gamma)^{2}}{\ell(Q)} \leq C h(\operatorname{diam}(\Gamma)) .
$$

Thus the lemma holds in this case.

Suppose now that $\Gamma \not \subset 2 Q$. From the fact that $\Gamma \cap Q \neq \varnothing$, we deduce

$$
\operatorname{diam}(\Gamma \cap 2 Q) \geq \frac{\ell(Q)}{2} .
$$

Let $z_{Q}$ be the center of $Q$. Notice that the ball $B\left(z_{Q}, 2 \ell(Q)\right)$ contains $\operatorname{supp}(\varphi)$. We consider the curve $\widetilde{\Gamma}:=\partial\left(B\left(z_{Q}, 2 \ell(Q)\right) \cap \operatorname{Int}(\Gamma)\right)$. Since $V_{\varphi} f$ is holomorphic outside $B\left(z_{Q}, 2 \ell(Q)\right)$, by Cauchy's theorem we have

$$
\int_{\Gamma} V_{\varphi} f(z) d z=\int_{\widetilde{\Gamma}} V_{\varphi} f(z) d z
$$

Therefore,

$$
\left|\int_{\Gamma} V_{\varphi} f(z) d z\right| \leq C\left\|V_{\varphi} f\right\|_{\infty} \mathcal{H}^{1}(\widetilde{\Gamma})
$$

Recall that from the identity

$$
V_{\varphi} f(z)=\frac{1}{\pi} \int \frac{f(z)-f(\xi)}{z-\xi} \bar{\partial} \varphi(\xi) d \mathcal{H}^{2}(\xi)
$$

one easily infers that $\left\|V_{\varphi} f\right\|_{\infty} \leq C \omega_{f}(\ell(Q))=C h(\ell(Q)) / \ell(Q)$. On the other hand, from the Ahlfors regularity of $\Gamma$, it easily follows that $\mathcal{H}^{1}(\widetilde{\Gamma}) \leq C \ell(Q)$. Thus, $\left|\int_{\Gamma} V_{\varphi} f(z) d z\right| \leq C h(\ell(Q))$ and so the lemma also holds in this situation, by $(26)$.

5.4. Proof of Theorem 4. Let $\Omega$ be the open set containing $E$ described in Lemma 5 , and $\Omega=\bigcup_{i \in I} Q_{i}$ the corresponding Whitney decomposition into squares $Q_{i}$. We consider the same family of $C^{\infty}$ functions $\varphi_{i}, i \in I$, used in the proof of the implication (b) $\Rightarrow$ (c) of Theorem 1 , with $\operatorname{supp}\left(\varphi_{i}\right) \subset \frac{3}{2} Q_{i}$, and also the same finite subfamily of squares $Q_{j}, j \in J \subset I$ (recall that these are the squares such that $\left.2 Q_{j} \cap E \neq \varnothing\right)$. Arguing as in the proof of $(\mathrm{b}) \Rightarrow(\mathrm{c})$, we have again

$$
\int_{\partial G} f(z) d z=\sum_{j \in J} \int_{\partial G} V_{\varphi_{j}} f(z) d z
$$

Let $J_{1} \subset J$ be the set of indices such that $2 Q_{j} \cap \partial G=\varnothing$ if $j \in J_{1}$, and $J_{2}=J \backslash J_{1}$. By Lemma 3 and the definition of $\gamma^{h}$, for $j \in J_{1}$ we have

$$
\left|\int_{\partial G} V_{\varphi_{j}} f(z) d z\right| \leq C \gamma^{h}\left(E \cap 2 Q_{j}\right),
$$


since $E \cap 2 Q_{i} \subset G$ and $C^{-1} V_{\varphi_{j}} f \in A^{h}\left(E \cap 2 Q_{j}\right)$, for some constant $C$. Therefore, by Lemma 5 and the fact that $\gamma^{h} \approx \gamma_{+}^{h}$,

$$
\left|\sum_{j \in J_{1}} \int_{\partial G} V_{\varphi_{j}} f(z) d z\right| \leq C \sum_{j \in J_{1}} \gamma^{h}\left(E \cap 2 Q_{j}\right) \leq C \gamma^{h}(E) .
$$

Now we have to deal with the squares $Q_{j}$ such that $2 Q_{j} \cap \partial G=\varnothing$. Let $\Gamma_{k}, k \in K$, denote the family of disjoint closed Jordan curves such that $\partial G=\bigcup_{k \in K} \Gamma_{k}$. Using Lemma 6 and the defnition of $\gamma^{h}$, for $j \in J_{2}$, we have

$$
\begin{aligned}
\left|\int_{\partial G} V_{\varphi_{j}} f(z) d z\right| & \leq \sum_{k: \Gamma_{k} \cap 2 Q_{j} \neq \varnothing}\left|\int_{\Gamma_{k}} \cdots\right|+\left|\sum_{k: \Gamma_{k} \cap 2 Q_{j}=\varnothing} \int_{\Gamma_{k}} \ldots\right| \\
& \leq C \sum_{k: \Gamma_{k} \cap 2 Q_{j} \neq \varnothing} h\left(\operatorname{diam}\left(\Gamma_{k} \cap 4 Q_{j}\right)\right)+C \gamma^{h}\left(\left[E \cup G^{c}\right] \cap 2 Q_{j}\right) .
\end{aligned}
$$

To estimate the last term we use the (countable) semiadditivity of $\gamma^{h}$ and the fact that $\gamma^{h}(F) \leq C h(\operatorname{diam}(F))$ for any compact set $F \subset \mathbb{C}$ :

$$
\begin{aligned}
\gamma^{h}\left(\left[E \cup G^{c}\right] \cap 2 Q_{j}\right) & \leq C \gamma^{h}\left(E \cap 2 Q_{j}\right)+C \gamma^{h}\left(G^{c} \cap 2 Q_{j}\right) \\
& \leq C \gamma^{h}\left(E \cap 2 Q_{j}\right)+C \sum_{k: \Gamma_{k} \cap 2 Q_{j} \neq \varnothing} h\left(\operatorname{diam}\left(\Gamma_{k} \cap 4 Q_{j}\right)\right) .
\end{aligned}
$$

Therefore, by Lemma 5 again,

$$
\left|\int_{\partial G} f(z) d z\right| \leq C \sum_{j \in J_{2}} \sum_{k: \Gamma_{k} \cap 2 Q_{j} \neq \varnothing} h\left(\operatorname{diam}\left(\Gamma_{k} \cap 4 Q_{j}\right)\right)+C \gamma^{h}(E) .
$$

Our next objective consists of showing that the first term on the right hand side in the preceding inequality is bounded above by $\gamma^{h}(E)$. To this end, for each $j \in J_{2}$ and $k \in K$ such that $\Gamma_{k} \cap 2 Q_{j} \neq \varnothing$, we consider a square $P_{k}^{j} \subset 4 Q_{j}$ with side length $\operatorname{diam}\left(\Gamma_{k} \cap 4 Q_{j}\right) / 2$ such that $P_{k}^{j} \cap \Gamma_{k} \neq \varnothing$. By Vitali's covering theorem, there exists a subfamily $\left\{P_{n}^{m}\right\}_{(m, n) \in S}$ of the squares $P_{k}^{j}$ such that the squares $2 P_{n}^{m}$, $(m, n) \in S$, are pairwise disjoint, and any square $P_{k}^{j}$ is contained in some square $6 P_{n}^{m}$, with $(m, n) \in S$. Now we consider the measure

$$
\mu:=\sum_{(m, n) \in S} \frac{h\left(\ell\left(P_{n}^{m}\right)\right)}{\mathcal{H}^{2}\left(P_{n}^{m}\right)} \mathcal{H}_{\mid P_{n}^{m}}^{2} .
$$

Observe that $\operatorname{supp}(\mu) \subset \Omega$. We claim that $\mu$ satisfies

$$
\begin{gathered}
\mu(\Omega) \geq C^{-1} \sum_{j \in J_{2}} \sum_{k: \Gamma_{k} \cap 2 Q_{j} \neq \varnothing} h\left(\operatorname{diam}\left(\Gamma_{k} \cap 4 Q_{j}\right)\right), \\
\mu(B(z, r)) \leq C h(r) \quad \text { for all } z \in \mathbb{C}, r>0,
\end{gathered}
$$

and also that the Cauchy integral operator $\mathcal{C}_{\mu}$ is bounded on $L^{2}(\mu)$, with constants depending only on the norm of the Cauchy integral operator $\mathcal{C}_{\mathcal{H}^{1} \mid \partial G}$ on $L^{2}\left(\mathcal{H}_{\mid \partial G}^{1}\right)$. Observe that using the characterization of $\gamma^{h}$ in terms of the $L^{2}$ norm of the Cauchy integral operator in (24), from our claims we deduce

$$
\sum_{j \in J_{2}} \sum_{k: \Gamma_{k} \cap 2 Q_{j} \neq \varnothing} h\left(\operatorname{diam}\left(\Gamma_{k} \cap 4 Q_{j}\right)\right) \leq C \mu(\Omega) \leq C \gamma^{h}(E),
$$

and then, by (29), the theorem follows. 
Let us see that (30) holds. Notice that for $j \in J_{2}$, and $k$ such that $\Gamma_{k} \cap 2 Q_{j} \neq \varnothing$, we have

$$
\ell\left(P_{k}^{j}\right)=\frac{1}{2} \operatorname{diam}\left(\Gamma_{k} \cap 4 Q_{j}\right) \leq C \mathcal{H}^{1}\left(\Gamma_{k} \cap 4 Q_{j}\right) .
$$

Taking into account that $h(r) / r$ is non decreasing, if $2 P_{k}^{j} \subset 6 P_{n}^{m}$, we get

Therefore,

$$
\begin{aligned}
h\left(\operatorname{diam}\left(\Gamma_{k} \cap 4 Q_{j}\right)\right) & \leq C h\left(\ell\left(P_{k}^{j}\right)\right) \leq C h\left(3 \ell\left(P_{n}^{m}\right)\right) \frac{\ell\left(P_{k}^{j}\right)}{3 \ell\left(P_{n}^{m}\right)} \\
& \leq C h\left(\ell\left(P_{n}^{m}\right)\right) \frac{\mathcal{H}^{1}\left(\Gamma_{k} \cap 4 Q_{j}\right)}{\ell\left(P_{n}^{m}\right)} .
\end{aligned}
$$

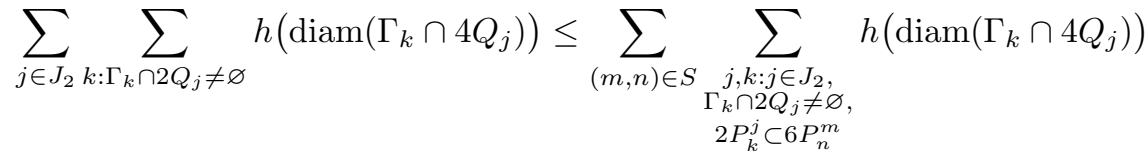

$$
\begin{aligned}
& \leq C \sum_{(m, n) \in S} \sum_{\substack{j, k: j \in J_{2}, 2 P_{k}^{j} \subset 6 P_{n}^{m}}} h\left(\ell\left(P_{n}^{m}\right)\right) \frac{\mathcal{H}^{1}\left(\Gamma_{k} \cap 4 Q_{j}\right)}{\ell\left(P_{n}^{m}\right)}
\end{aligned}
$$

To estimate the last sum notice that, by construction, if $2 P_{k}^{j} \subset 6 P_{n}^{m}$, then we have

$$
\Gamma_{k} \cap 4 Q_{j} \subset 6 P_{k}^{j} \subset 18 P_{n}^{m} .
$$

Thus, using the finite overlap of the Whitney squares $Q_{j}$, for each fixed $(m, n) \in S$, we obtain

$$
\begin{aligned}
\sum_{\substack{j, k: j \in J_{2}, 2 P_{k}^{j} \subset 6 P_{n}^{m}}} \mathcal{H}^{1}\left(\Gamma_{k} \cap 4 Q_{j}\right) & \leq \sum_{k} \sum_{j \in J_{2}} \mathcal{H}^{1}\left(18 P_{n}^{m} \cap \Gamma_{k} \cap 4 Q_{j}\right) \\
& \leq C \sum_{k} \mathcal{H}^{1}\left(18 P_{n}^{m} \cap \Gamma_{k}\right)=\mathcal{H}^{1}\left(18 P_{n}^{m} \cap \partial G\right) \leq C \ell\left(P_{n}^{m}\right) .
\end{aligned}
$$

If we plug this estimate into (32), (30) follows:

$$
\sum_{j \in J_{2}} \sum_{k: \Gamma_{k} \cap 2 Q_{j} \neq \varnothing} h\left(\operatorname{diam}\left(\Gamma_{k} \cap 4 Q_{j}\right)\right) \leq C \sum_{(m, n) \in S} h\left(\ell\left(P_{n}^{m}\right)\right)=C \mu(\Omega) .
$$

Now we will prove (31). Observe that it is enough to prove it for $z \in \operatorname{supp}(\mu)$. So take a fixed $P_{n}^{m},(m, n) \in S$, and $z \in P_{n}^{m}$. Suppose first that $r \leq \ell\left(P_{n}^{m}\right) / 2$. Since $2 P_{n}^{m}$ does not intersect any other square $P_{n^{\prime}}^{m^{\prime}},\left(m^{\prime}, n^{\prime}\right) \in S$, and on $2 P_{n}^{m} \mu$ coincides with the Lebesgue measure times $h\left(\ell\left(P_{n}^{m}\right)\right) / \ell\left(P_{n}^{m}\right)^{2}$, we have

$$
\mu(B(z, r)) \leq C \frac{h\left(\ell\left(P_{n}^{m}\right)\right)}{\ell\left(P_{n}^{m}\right)^{2}} r^{2} .
$$

From the property (23) of the function $h$, setting $\lambda=\ell\left(P_{n}^{m}\right) /(2 r),(31)$ follows (for $\left.0<r \leq \ell\left(P_{n}^{m}\right) / 2\right)$.

Assume now that $r>\ell\left(P_{n}^{m}\right) / 2$. We set $T=\left\{(p, q) \in S: P_{q}^{p} \cap B(z, r) \neq \varnothing\right\}$. Since $2 P_{n}^{m} \cap 2 P_{q}^{p}=\varnothing$ if $(m, n) \neq(p, q)$, we have $r \geq \ell\left(P_{q}^{p}\right) / 2$ for any $(p, q) \in T$. So there exists some constant $C_{12}$ such that

$$
\bigcup_{(p, q) \in T} P_{q}^{p} \subset B\left(z, C_{12} r\right) .
$$


Recall that

$$
\ell\left(P_{q}^{p}\right)=\frac{1}{2} \operatorname{diam}\left(\Gamma_{q} \cap 4 Q_{p}\right) \leq C \mathcal{H}^{1}\left(\Gamma_{q} \cap 4 Q_{p}\right),
$$

because $\Gamma_{q} \cap 2 Q_{p} \neq \varnothing$. Then, by the finite overlap of the squares $4 Q_{p}, p \in J_{2}$ (recall that the $Q_{p}$ 's are Whitney squares), we get

$$
\begin{aligned}
\sum_{(p, q) \in T} \ell\left(P_{q}^{p}\right) & \leq C \sum_{(p, q) \in T} \mathcal{H}^{1}\left(\Gamma_{q} \cap 4 Q_{p}\right) \leq C \sum_{(p, q) \in S} \mathcal{H}^{1}\left(B\left(z, C_{12} r\right) \cap \Gamma_{q} \cap 4 Q_{p}\right) \\
& \leq C \mathcal{H}^{1}\left(B\left(z, C_{12} r\right) \cap \partial G\right) \leq C r .
\end{aligned}
$$

Taking into account that $h(t) / t$ is non decreasing, we obtain

$$
\begin{aligned}
\mu(B(z, r)) & \leq \sum_{(p, q) \in T} h\left(\ell\left(P_{q}^{p}\right)\right)=\sum_{(p, q) \in T} \frac{h\left(\ell\left(P_{q}^{p}\right)\right)}{\ell\left(P_{q}^{p}\right)} \ell\left(P_{q}^{p}\right) \\
& \leq \frac{h(2 r)}{2 r} \sum_{(p, q) \in T} \ell\left(P_{q}^{p}\right) \leq C h(2 r) \leq C h(r) .
\end{aligned}
$$

The $L^{2}(\mu)$ boundedness of the Cauchy integral operator $\mathcal{C}_{\mu}$ follows from the $L^{2}$ boundedness of the Cauchy integral operator on $L^{2}\left(\mathcal{H}_{\mid \partial G}^{1}\right)$ by comparison, and using the linear growth of $\mu$ (which is a consequence of (31)). We leave the details for the reader.

Remark 7 The implication (b) $\Rightarrow$ (c) of Theorem 1 can be proved using arguments closer to the ones used to prove Theorem 4 than the ones in Subsection 4.2. More precisely, one can obtain a variant of Lemma 6 suitable for $L^{\infty}$ functions, and use it to estimate $\int_{\partial G} V_{\varphi_{j}} f(z) d z$ for $j \in J_{2}$. Recall that, instead, in Subsection 4.2 the integral $\int_{\partial G} V_{\varphi_{j}} f(z) d z$ was estimated with the help of some auxiliary function $g_{j}$ holomorphic outside some disk $D_{j}$.

\section{Proof of the implication (c) $\Rightarrow$ (a)}

We want to prove that if the estimate of the integral (3) holds, then for any set $F \subset \partial G$ we have

$$
\gamma(F) \geq C^{-1} \mathcal{H}(F)
$$

Given $n \geq 1$, let $\delta>0$ be such that $\gamma\left(U_{\delta}(F)\right) \leq \gamma(F)+1 / n$. Using Vitali's covering theorem, it is easy to check that there exists a finite family of pairwise disjoint balls $\left\{B\left(x_{j}, r_{j}\right)\right\}_{j \in J}$ with $x_{j} \in \partial G, B\left(x_{j}, r_{j}\right) \subset U_{\delta}(F)$, such that

$$
\mathcal{H}^{1}(F) \leq C \mathcal{H}^{1}\left(\bigcup_{j \in J} B\left(x_{j}, r_{j}\right) \cap F\right) .
$$

Moreover, using well known elementary properties of the 1-dimensional Hausdorff measure, since $\mathcal{H}^{1}(F)<\infty$, we may assume that the radii $r_{j}$ are small enough so that $\mathcal{H}^{1}\left(B\left(x_{j}, r_{j}\right) \cap F\right) \leq 3 r_{j}$, and then we have

$$
\mathcal{H}^{1}(F) \leq C \sum_{j \in J} r_{j} .
$$

Given any $\varepsilon$, with $0<\varepsilon \leq \min _{j \in J} r_{j} / 10$, for each fixed $j \in J$ there are points $a_{j}^{+}, b_{j}^{+}, a_{j}^{-}, b_{j}^{-} \in \mathbb{C}$ which satisfy the following properties:

(a) $a_{j}^{+}, b_{j}^{+} \in B\left(x_{j}, r_{j} / 3\right) \cap G$ and $a_{j}^{-}, b_{j}^{-} \in B\left(x_{j}, r_{j} / 3\right) \cap \mathbb{C} \backslash \bar{G}$, 
(b) $r_{j} / 3 \leq\left|a_{j}^{+}-b_{j}^{+}\right|$and $r_{j} / 3 \leq\left|a_{j}^{-}-b_{j}^{-}\right|$,

(c) $\left|a_{j}^{+}-a_{j}^{-}\right| \leq \varepsilon$ and $\left|b_{j}^{+}-b_{j}^{-}\right| \leq \varepsilon$, and

(d) there exists a simple rectifiable arc $\sigma_{j}^{+}$contained in $B\left(x_{j}, r_{j} / 2\right) \cap G$ whose endpoints are $a_{j}^{+}, b_{j}^{+}$, and another simple rectifiable arc $\sigma_{j}^{-}$contained in $B\left(x_{j}, r_{j} / 2\right) \cap \mathbb{C} \backslash \bar{G}$ whose endpoints are $a_{j}^{-}, b_{j}^{-}$.

Notice that from (33) and the properties (a) and (b), we deduce

$$
\mathcal{H}^{1}(F) \leq C \sum_{j \in J}\left|a_{j}^{+}-b_{j}^{+}\right| \approx \sum_{j \in J}\left|a_{j}^{-}-b_{j}^{-}\right| .
$$

For each $j \in J$, consider the univalued branch of the function

$$
f_{j}^{+}(z):=\frac{-\left(\overline{b_{j}^{+}-a_{j}^{+}}\right)^{2}}{\left|b_{j}^{+}-a_{j}^{+}\right|^{3}}\left(z-\frac{a_{j}^{+}+b_{j}^{+}}{2}+\sqrt{\left(z-a_{j}^{+}\right)\left(z-b_{j}^{+}\right)}\right),
$$

which is holomorphic on $\mathbb{C} \backslash \sigma_{j}^{+}$and vanishes at $\infty$. Let also $f_{j}^{-}$be the univalued branch, holomorphic on $\mathbb{C} \backslash \sigma_{j}^{-}$, of the analogous function $f_{j}^{-}$, vanishing at $\infty$, defined like $f_{j}^{+}$, interchanging $a_{j}^{+}, b_{j}^{+}$by $a_{j}^{-}, b_{j}^{-}$. Then we have

$$
\left\|f_{j}^{+}\right\|_{\infty}=\left\|f_{j}^{-}\right\|_{\infty} \leq C_{13}
$$

and

$$
\int_{\partial G} f_{j}^{+}(z) d z=\left(f_{j}^{+}\right)^{\prime}(\infty)=\left|a_{j}^{+}-b_{j}^{+}\right|, \quad \int_{\partial G} f_{j}^{-}(z) d z=0 .
$$

We set

$$
f:=\sum_{j \in J}\left(f_{j}^{+}-f_{j}^{-}\right)
$$

It is easy to check that

$$
\left\|f_{j}^{+}-f_{j}^{-}\right\|_{\infty, \mathbb{C} \backslash B\left(x_{j}, r_{j}\right)}=C(\varepsilon) \rightarrow 0 \quad \text { as } \varepsilon \rightarrow 0,
$$

Thus, we deduce

$$
\|f\|_{\infty} \leq 2 C_{13}+C(\varepsilon) \cdot \# J .
$$

If we choose $\varepsilon$ such that $C(\varepsilon) \leq 1 / \# J$, we have $\|f\|_{\infty} \leq 2 C_{13}+1$, and then using (34), (35), and (3), we get

$$
\begin{aligned}
\mathcal{H}^{1}(F) & \leq C \sum_{j \in J}\left|a_{j}^{+}-b_{j}^{+}\right|=\int_{\partial G} \sum_{j \in J} f_{j}^{+}(z) d z=\int_{\partial G} f(z) d z \\
& \leq C \gamma\left(\bigcup_{j \in J} \sigma_{j}^{+}\right) \leq C \gamma\left(U_{\delta}(F)\right) \leq C\left(\gamma(F)+\frac{1}{n}\right) .
\end{aligned}
$$

Since this estimate holds for any $n \geq 1$, we are done.

\section{Proof of the implication (d) $\Rightarrow$ (a)}

The proof is analogous to the one of the implication $(\mathrm{c}) \Rightarrow(\mathrm{a})$. We only have to change the function $f$ defined in (36) by a continuous function $\widetilde{f}$ which coincides with $f$ outside some small neighborhood of $\bigcup_{j \in J}\left(\sigma_{j}^{+} \cup \sigma_{j}^{-}\right)$, so that $f=\tilde{f}$ on $\partial G$, and then we apply the estimate of the integral (4) for $\tilde{f}$. 


\section{REFERENCES}

[Dd] G. David, Opérateurs intégraux singuliers sur certaines courbes du plan complexe, Ann. Sci. École Norm. Sup. (4) 17 (1984), no. 1, 157-189.

[De] A. M. Davie, Analytic capacity and approximation problems, Trans. Amer. Math. Soc. 171 (1972), 409-444.

[Ga] T. Gamelin, Uniform Algebras, Prentice Hall, Englewood Cliffs N.J., 1969.

[Me1] M. S. Melnikov, Estimate of the Cauchy integral over an analytic curve, (Russian) Mat. Sb. 71(113) (1966), 503-514. Amer. Math. Soc. Translation 80(2) (1969),243-256.

[Me2] M. S. Melnikov, Analytic capacity: discrete approach and curvature of a measure, Sbornik: Mathematics 186(6) (1995), 827-846.

[MV] M. S. Melnikov and J. Verdera, A geometric proof of the $L^{2}$ boundedness of the Cauchy integral on Lipschitz graphs, Internat. Math. Res. Notices (1995), 325-331.

[NTV1] F. Nazarov, S. Treil and A. Volberg, Weak type estimates and Cotlar inequalities for Calderón-Zygmund operators in nonhomogeneous spaces, Int. Math. Res. Not. 9 (1998), 463-487.

[NTV2] F. Nazarov, S. Treil and A. Volberg, Accretive system Tb-theorems on nonhomogeneous spaces, Duke Math. J. 113 (2002), 259-312.

[Pa] P. V. Paramonov, Some new criteria for the uniform approximability of functions by rational fractions, (Russian) Mat. Sb. 186 (1995), no. 9, 97-112. Translation in Sb. Math. 186 (1995), no. 9, 1325-1340.

[To1] X. Tolsa, $L^{2}$-boundedness of the Cauchy integral operator for continuous measures, Duke Math. J. 98(2) (1999), 269-304

[To2] X. Tolsa, A proof of the weak $(1,1)$ inequality for singular integrals with non doubling measures based on a Calderón-Zygmund decomposition, Publ. Mat. 45:1 (2001), 163-174.

[To3] X. Tolsa, On the analytic capacity $\gamma_{+}$, Indiana Univ. Math. J. 51(2) (2002), 317-344.

[To4] X. Tolsa, Painlevés problem and the semiadditivity of analytic capacity, Acta Math. 190:1 (2003), 105-149.

[To5] X. Tolsa, The semiadditivity of continuous analytic capacity and the inner boundary conjecture. Preprint (2002). To appear in Amer. J. Math.

[Ve] J. Verdera. On the T(1)-theorem for the Cauchy integral. Ark. Mat. 38 (2000), 183-199.

[Vi1] A. G. Vitushkin, Estimate of the Cauchy integral, (Russian) Mat. Sb. 71 (113) (1966), $515-534$.

[Vi2] A. G. Vitushkin, The analytic capacity of sets in problems of approximation theory, Uspeikhi Mat. Nauk. 22(6) (1967), 141-199 (Russian); in Russian Math. Surveys 22 (1967), 139-200.

Departament de Matemàtiques, Universitat Autònoma de Barcelona, Spain

E-mail address: melnikov@mat.uab.es

ICREA And Departament de Matemàtiques, Universitat Autònoma de Barcelona, SPAIN

E-mail address: xtolsa@mat.uab.es 